Farley, S, Durodola, J, Fellows, N and Hernandez-Gomez, L) High-resolution non-destructive evaluation of defects using artificial neural networks and wavelets.

Farley, S, Durodola, J, Fellows, N and Hernandez-Gomez, L (2012) High-resolution non-destructive evaluation of defects using artificial neural networks and wavelets. NDT\& E International, 52

(November). pp. 69-75.

This version is available: https://radar.brookes.ac.uk/radar/items/e78c0917-b519-4b15-8384-4f2f0c60d347/1/

Available on RADAR: June 2013

Copyright (C) and Moral Rights are retained by the author(s) and/ or other copyright owners. A copy can be downloaded for personal non-commercial research or study, without prior permission or charge. This item cannot be reproduced or quoted extensively from without first obtaining permission in writing from the copyright holder(s). The content must not be changed in any way or sold commercially in any format or medium without the formal permission of the copyright holders.

This document is the postprint of the journal article. Some differences between the published version and this version may remain and you are advised to consult the published version if you wish to cite from it. 


\title{
High resolution nondestructive evaluation of defects using artificial neural networks and wavelets
}

\author{
S J Farley ${ }^{\mathrm{a}}$, J F Durodola ${ }^{\mathrm{a} *}$, N A Fellows ${ }^{\mathrm{a}}$ and L H Hernández-Gómez ${ }^{\mathrm{b}}$ \\ Oxford Brookes University, Faculty of Technology, Design and Environment, Wheatley \\ Campus, Oxford OX33 1HX, UK . \\ Instituto Politécnico Nacional, ESIME SEPI Edificio 5, 3er piso, Unidad Profesional Adolfo \\ López Mateos, colonia Lindavista, Gustavo A Madero, 07738 México, D. F. México ${ }^{\text {b }}$
}

\begin{abstract}
This paper presents artificial neural networks (ANN) and wavelet analysis as methods that can assist high resolution of multiple defects in close proximity in components. Without careful attention to analysis, multiple defects can be mis-interpreted as single defects and with the possibility of significantly underestimated sizes. The analysis in this work focussed on A-scan type ultrasonic signal. Amplitudes corresponding to the sizes of two defects as well as the phase shift parameter representing the distance between them were determined. The results obtained demonstrates very good correlation for sizes and distances respectively even in cases involving noisy signal data.
\end{abstract}

Keywords multi defects, ultrasonic, artificial neural networks, wavelets, non destructive evaluation. 


\section{Introduction}

Ultrasonic methods are used routinely for the detection of flaws during non-destructive evaluation (NDE) of components. In this process, the ultrasonic wave transmitted to the component travels and reflects back at defects and discontinuities such as voids, cracks and inclusions. In the pulse echo method, the size and distance of defects are determined by the amplitude of the reflected wave and the time of flight (TOF) i.e. the time it takes for the incident wave to travel to the defect and for the reflected wave to return to the probe. Data logging allows the time of flight to be determined and calibration methods are used to determine the size of the defect from the amplitude of the reflected wave. Interpretation of logged data is carried out using various linear and nonlinear signal processing methods. These include the use of cross-correlation, convolution, blind sources separation, split spectral processing and wavelet methods. In these processes, the determination of required parameters such as location, size and shape of defects is adversely affected by the noise in the signal and sometimes by interfering features such as defects in close proximity, boundaries in thin layered components e.g. microelectronic circuit boards, and multiple thin layers in composite materials. Although artificial neural network and wavelet methods have been used to explore signal processing for NDE in general, there have been little or no attempt to consider its use for the intricate cases highlighted in this paper.

A lot of effort has been applied in the literature to the development of methods for the analysis of thin and multilayered composites, see for example reference [1]. This paper focuses on the use of artificial neural network methods for the resolution of closely spaced defects as very little has been reported in the literature on how to deal with this characterisation problem. Without careful attention to data processing multiple defects can be mis-interpreted as single defects and the size can be significantly underestimated. This 
problem arises because of the complex form of the signal that is produced when the reflected waves from close defects overlap. The effects of various factors such as the relative sizes of defects, the order in which they occur in the component, the closeness of the interfering defects and signal digitisation and sampling methods on the accuracy of predictions are analysed. The results obtained generally demonstrate very good ability of ANN and wavelets to characterise multiple defects in components.

\section{Theory}

The theory presented in this section aims to highlight the various principles and relationships underlining the ultrasonic A-scan, ANN and wavelet analyses carried out in the paper. The interest in this work is to present artificial neural network and wavelets as a method that can be used to characterise the presence of defects $a$ and $b$, Fig 1, in terms of location and size. These parameters can be determined by using a pulse echo ultrasonic non destructive evaluation method. The location is determined from the relationship between time, distance and the speed of sound in the material and the size depends on the magnitude of the amplitude of the reflected wave from the defects. The analysis considered in this work concerns the case of reflection of pressure waves. In general wave propagation in a continuum is subject to complicating factors such as the effects of mixture of modes of motion, multi-axiality of internal microstructural grains, and grain and component boundaries. These factors lead to internal damping and together with component shape lead to wave dispersion. These problems are usually resolved by using appropriate hardware and calibration methods for known defect characteristics. The resolution and characterisation considered in this work are based on the availability of both hardware and the type of analysis carried out in this work validated by experiment. 


\subsection{Overlapping ultrasonic pulse reflections}

Figure 1 shows an illustration of the superposition of waves that are reflected from two close defects $a$ and $b$ having different sizes and separated by a small distance $e$. The reflections will overlap if the distance $e$ is less than half the length of the pulse emitted by the transducer i.e. if $e<n \lambda_{w} / 2$. In this, $n$ is the number of wavelengths and $\lambda_{w}$ is the wavelength of the signal that is emitted by the ultrasonic probe. The value of $n$ used is 3.4 which is an estimate from the plot of a real signal shown in reference [2]. For a material such as steel, the distance $e$ is in the range $3 \mathrm{~mm}$ to $100 \mathrm{~mm}$ for ultrasonic probes with central frequencies ranging between $100 \mathrm{kHz}$ to $5 \mathrm{MHz}$. Equations (1), (2) and (3) give a description of the reflected superposed wave.

$$
\begin{gathered}
u_{r}(t)=A \sin (\omega t) E_{a}(t) H_{a}(t)+B \sin (\omega t-\tau) E_{b}(t) H_{b}(t) \\
E_{i}(t)=\frac{1}{\sigma \sqrt{2 \pi}} \exp \left[\frac{\left(t-\mu_{i}\right)^{2}}{2 \sigma^{2}}\right] \\
H_{i}(t)=H\left(\omega t-S_{i}\right)
\end{gathered}
$$

where $u_{r}(t)$ is the displacement at a particular location in a component at a time $t$ and subscript $i=a$ or $b$ relate to the reflections from defects $a$ and $b ; \mu_{a}=n \lambda_{w} / 2, \mu_{b}=\tau+n \lambda_{w} / 2, S_{a}=n \lambda_{w}$ and $S_{b}=\tau+n \lambda_{w} . A$ and $B$ are the amplitudes that correspond to the sizes of the defects and the phase shift $\tau$ corresponds to the distance between them. The value of $\sigma$ was taken to be 3.5 to produce similar signal as in reference [2]. The first and second parts of the right hand side of the equation (1) represent the reflected signal from the first and second defects encountered. The Heaviside step function $H$ () (which is equal to 0 when the arguement in the bracket is $\leq 0$ but is otherwise $=1$ when the argument is $>0$ ). The function delimits the intervals over which the two reflected signals occur in the time frame. There are other descriptions of single burst of signal functions, an example can be seen in [3]. 
A particular close defect problem will be one out of many infinitely possible combinations of the central amplitudes $A$ and $B$ and the phase shift $\tau$. These parameters characterise the size of the two defects and distance $e$ between them. When an ultrasonic probe sends pulses into the component, the reflections from the defect inherently contain information that can be used for the determination of the parameters even in the intricate cases of interfering defects involving discontinuities in the signal. Rule based algorithms will be cumbersome if not impractical for the interpretation of these types of signals. Also cross-correlation methods involving the convolution of original and reflected signals will be inadequate for the determination of all the characterising parameters required because the original and reflected signals are of two different forms at least in the overlapping section. Artificial neural network and wavelets are useful methods for the solution of these types of problems. They have both pattern recognition and non-linear function approximation capabilities. ANN methods are known to be useful for interpretation of data even when there is noise in the input data [4]. It should be hoighlighted that only defects that are orthogonal to the beam or nearly so can be identified by an A-scan method.

\section{Artificial neural network and wavelet approach}

The implementation of the artificial neural network analysis in this work used three layers of neurons which is generally accepted as sufficient to represent any non-linear relationship [5]. The data input represents the combined overlapping reflected pulse. The details of the number of input neurons are given section 3.2 where the different combinations of ANN and wavelets used are presented. The number of neurons in the output layer was three representing the amplitudes $A$ and $B$ and the phase shift $\tau$. Although the number of neurons in the internal layer has commonly been determined by trial and error, it is now empirically accepted to be equal to the average of the number of neurons in the output and the input layers [6]. 
Each internal and output neuron $i$ receives a weighted sum $x_{i}$ of input values $x_{j}$ from the preceding neurons $j$ according to equation (4). The output from the neuron $y_{i}=f\left(x_{i}\right)$ were sigmoidal and linear functions as given in equations (5) and (6) for the internal and output neurons respectively;

$$
\begin{gathered}
x_{i}=\sum_{j} w_{i j} x_{j}-\theta_{i} \\
y_{i}=\frac{1}{1+\exp \left(-\sigma x_{i}+\theta_{i}\right)} \\
y_{i}=x_{i}+\theta_{i}
\end{gathered}
$$

$w_{i j}$ is the weight connecting neuron $j$ to neuron $i$; $\theta_{i}$ is the bias at neuron $i$. An error backpropagation method was used for the training process which updated the weights $w_{i j}$. Various backpropagation algorithms have been devised for the training of networks. The primary method used in this analysis was based on a variation of the + Rprop algorithm which is known to have excellent convergence characteristics [7]. For research flexibility purposes, the implementation was carried out using a set of in house routines developed in a MATLAB [8] environment. The parameters required for the optimal convergence of the training have been identified for most problems and are not dependent on trial and error. A heuristic guideline was used to set the number of cases required for the neural network training to be about ten times the total sum of all the neurons in the network [6].

\subsection{Wavelet analysis}

Fast discrete wavelet analysis was carried out with the aims for feature extraction and for denoising purposes. The objective was to take advantage of the possible benefits of coupling this to the neural network method for the defect characterisation analysis. Features were extracted by using wavelet decomposition on the signal $u_{r}(t)$ as expressed in equation (7) to 
obtain the approximation $a(L, k)$ and detail $d(j, k)$ wavelet coefficients given in equations (8) and (9) respectively [9-12].

$$
\begin{gathered}
u_{r}(t)=\sum_{k} a(L, k) \phi\left(2^{-L} t-k\right)+\sum_{j=1}^{L} \sum_{k} d(j, k) \psi\left(2^{-j} t-k\right) \\
a(L, k)=2^{-L / 2} \sum_{t} u_{r}(t) \phi\left(2^{-L} t-k\right) \\
d(j, k)=2^{-j / 2} \sum_{t} u_{r}(t) \psi\left(2^{-j} t-k\right)
\end{gathered}
$$

The mother and scaling wavelet $\psi$ and $\phi$ respectively are quadrature mirror filters which satisfy orthogonality and multi-resolution requirements $[13,14] . L$ is the maximum level of resolution specified and $k$ is the index for localisation of the wavelet during translation on the time scale. The Daubechies DAUB4 wavelet transform scheme [13] was used to obtain the approximation and detail wavelet coefficients for the reflected ultrasonic signal. The details of the feature extraction used for the of the ANN are given in section 3.2.

\subsection{Combination of ANN and wavelet analysis}

ANN and wavelet analysis were applied singly or in combination in two ways denoted as methods $M 1$ and $M 2$. Feature extractions considered the options presented in references [1519]. Method $M 1$ used all the maximum $128 u_{r}(t)$ data points available as feature for the ANN training [16]. In method $M 2$, the best fitting wavelet decomposition coefficients were used as the feature for training the ANN [16]. This was obtained as the approximation coefficients for the first level decomposition given by equation (8). The number of coefficients in this case was 67.

The number of patterns used for the ANN training was 2000 and convergence occurred at about 2000 epochs of iteration. For all training processes, $80 \%$ of the pattern data cases was used for the training, $10 \%$ was used for validation and the last $10 \%$ was used for the 
assessment of the network. In all cases the known amplitude $A$ and $B$ and phase shift $\tau$ were used as the expected output for the network training.

\section{Background experimental testing}

In practical testing, the amplitudes $A$ and $B$ in equation (1) registered by an ultrasonic probe are affected by several factors such as the contact surface roughness of the material, distance of defects to probe and orientation of defect to beam, material grain structure and size, and component geometry. These factors generally result in signal scattering, diffraction, mode conversion and damping. Attenuation which is primarily due to damping, scattering, beam spreading, and signal frequency is generally accounted for in practical applications by the use of distance amplitude correction (DAC) curves. In the background study for this work [20] DAC curves were generated for a $200 \times 100 \times 10 \mathrm{~mm}$ mild steel samples and a $5 \mathrm{MHz}$ central frequency Socomate NDT transducer used for the experiment [21]. Three hole defect diameter sizes $d=1,2$ and $3 \mathrm{~mm}$ at 13 depth locations in steps of $5 \mathrm{~mm}$ in the range 70 to $140 \mathrm{~mm}$ were sampled for the construction of attaenuation curves, Figure 2. An approximate attenuation relationship $A=A_{o}$ d.exp ${ }^{-\alpha x}$ was developed using least square fit method which gave $A_{o}=248 \% / \mathrm{mm}$ and $\alpha=0.023 / \mathrm{mm}, d$ is the size of defect and $x$ is the distance from the probe. This relationship was used in equation (1) to account for attenuation in the signals used in the study.

\section{Results}

The wavelet and artificial neural networks described in the foregoing was tested using three main case studies. The first case concentrated on the ability of the methods $M 1$ and $M 2$ to determine the central amplitudes $A$ and $B$ and the phase shift parameter $\tau$ from the response composed by the reflections from two close defects as highlighted in equations (1) - (3). This 
aspect aimed to reveal the effect of the complexity of the form of the compounded reflected waves from the close defects on the ability of the methods to make a prediction. The second case considered the effect of the limits on the central amplitudes $A$ and $B$ and the phase shift $\tau$ on predictions. The third case considers the effect of noise on the parameter estimation problems for defect sizes and location.

\subsection{Amplitude and phase shift determination using methods $M 1$ and $M 2$.}

In this case, the amplitudes $A$ and $B$ were varied from zero to a nominal value of 2 while $\tau$ varied from 0 to the limit $n \lambda_{w} / 2$ which was about 11 radians in this study. Each of the parameters was randomly varied between the limits when the pattern cases for the training of the ANN were composed. The Latin Hypercube Sampling (LHS) [15] was used for the multivariate sampling to ensure more representative consideration of real variability.

Figure 3 shows the comparison of predicted against actual values for the two methods $M 1$ and M2. It can be seen that both methods generally show excellent correlation for the amplitude A. Very similar results were obtained for amplitude $B$ and are therefore not shown. The parameter estimation from the wavelet method $M 2$ appears as good as for case $M 1$ even though the former used only about half the data input (67) as the latter (128). The correlation for the phase shift $\tau$ although generally acceptable is not as good as for the amplitudes $A$ and $B$. It can be seen from the Figures $3(\mathrm{~b})$ and (d) that there appear to be more deviation from target values especially at about $\tau=\pi / 2$. A close examination also revealed that it was difficult to obtain good predictions for $\tau$ where an amplitude in the response function $u_{r}(t)$ was vanishingly small. This closeness to homogeneity will be testing for any method of signal analysis. The values of $A, B$ and $\tau$ for one such case were $1.4025,0.0005$ and 9.8883 respectively. It can be seen that second defect in this case has a very small amplitude 
$B=0.0005$; three to four orders of magnitude smaller than A. It is very difficult in these cases to detect where the discontinuity due to the phase shift starts or ends in the superposed signal. Although the corresponding amplitudes were closely predicted, the phase shift prediction deviated significantly from the target value. Care therefore needs to be taken in the interpretation of phase shifts where a defect size is vanishingly small.

\subsection{Effect of the range of parameters $A, B$ and $\tau$ on accuracy of ANN training}

This case attempts to detect the sensitivity of accuracy of prediction on the range of the parameters used in training. This is an essential factor to be considered in a multivariate experimental design problem [22]. The lower and upper limits considered for each of the amplitudes $A$ and $B$ were nominally set as 1 and 2 respectively. The lower limit set for the phase shift $\tau$ was $25 \%$ of the maximum limit of $n \lambda_{w} / 2$. The upper limit was set to the maximum value $n \lambda_{w} / 2$. In order to generate the sample data for the neural network training, each range was sampled using the LHS method as highlighted in section 4.1 above. The combination of limits considered in evaluating the methods $M 1$, and $M 2$ are shown in Table 1. The lower and upper limits are indicated by superscript symbols '-' and '+' respectively. The results of the studies were assessed by using the Pearson product-moment correlation coefficient, $R_{X, Y}$, equation (10) and the normalised root mean square deviation, $E_{n}$, equation

$$
\begin{aligned}
& R_{X, Y}=\frac{E\left[\left(X-\mu_{X}\right)\left(Y-\mu_{Y}\right)\right]}{\sigma_{X} \sigma_{Y}} \\
& E_{n}=E\left[(Y-X)^{2}\right]^{1 / 2} /(\hat{X}-\breve{X})
\end{aligned}
$$


where $X$ and $Y$ represent the predicted and actual values for $A, B$ or $\tau$ and $\mu, \sigma$ and $E$ denote statistical mean, standard deviation and expectation respectively. Both $R_{X, Y}$, and $E_{n}$ were determined for $A, B$ and $\tau$ in Table 1 to demonstrate the extent of correlation and accuracy respectively. $E_{n}$ presented in the Table 1 is the average value for the three parameters $A, B$ and $\tau$

It can be seen from the table that for each of the methods $M 1$ and $M 2$ predicted and actual values show high values of correlation for $A, B$ and $\tau$ for test cases 1 and 3 where the range of $\tau$ is at the lower limit. It can also be seen that the normalised root mean square deviation for these test cases are lower than for the cases 2 and 4 where the range was set at the higher limit. As indicated the training of the neural network carried out with the upper limit on the range of $\tau$ led to less accurate predictions. This shows high sensitivity to changes in $\tau$. This observation is predictable when the form of the response $u_{r}(t)$ in equations (1), (2) and (3) is considered. The phase shift $\tau$ is responsible for most of the complexities of the function. It is also responsible for the discontinuities represented by the Heaviside step function $H($ ).

\subsection{Effect of noise in data and sampling characteristics}

In order to assess the sensitivity of the procedure implemented in the study, different levels of experimental error were approximated by adding a white Gaussian noise to the original signal. The root mean square (rms) amplitude of the noise signal $A_{n}$ for different signal to noise ratio $\mathrm{S}_{\mathrm{NR}}$ in $\mathrm{dB}$ was obtained in terms of the signal root mean square amplitude $\mathrm{A}_{\mathrm{s}}$ by using the classical equation (12). The noise was added by applying the equation (13) at each data point.

$$
\begin{aligned}
& S_{N R}(d B)=10 \log _{10} \frac{A_{s}^{2}}{A_{n}^{2}} \\
& u_{r}^{\prime}(t)=u_{r}(t)+A_{n} \cdot \sim N(0,1)
\end{aligned}
$$


where the second term in (13) denotes the product of $A_{n}$ with a randomly seleted member of a standard normal distribution population with zero mean and variance $=1$ having the same size as the signal $\mathrm{u}_{\mathrm{r}} ; u_{r}^{\prime}(t)$ is the modified signal data which includes the added noise. Four signal to noise ratio levels $\mathrm{S}_{\mathrm{NR}}=7,1421$ and 30 representing very poor to excellent signals were considered. It was found helpful to apply a digital filter to the signals before using them to train the artificial neural network. A fourth order Butterworth low pass pass filter [8] with a cut-off frequency selected to minimise the root mean square difference between the raw and filtered signals was used. Figures 4(a) and (b) show two signals out of 2000 different combinations that were studied each with $7 \mathrm{~dB}$ signal to noise ratio. Figures (c) and (d) show the corresponding original signals and the signal obtained by using the Butterworth filter on the noisy signals. The root means square deviation between the original and the filtered signals are very small at 0.006 and $0.004 \%$ for the cases (c) and (d) respectively. There is nevertheless mismatch between the two signals that affect overall accuracy.

It can be seen from Table 2 and Figure 5 that the correlation between predicted and target values for the two methods is generally good although there is some scatter. The band lines included in the figure show that about $90 \%$ of predictions are within $10 \%$ deviation from the maximum value for the poorest signal case with $S_{N R}=7$. In the case of $S_{N R}=21$ corresponding value is about $97 \%$ for $\tau$ and $99 \%$ for $A$. Table 2 gives the level of correlation $R_{X, Y}$ and the normalised root mean square error when the signal included noise. Without filtering the deviation is worse especially for the very poor signal to noise ratio $S_{N R}=7$. Filtering the signal before using the wavelet method did not produce better results than direct application of ANN on the filtered data. 


\section{Discussion}

This paper focuses attention on the need to characterise multiple defects as a means to achieving higher resolution non-destructive evaluation of components. The types of problems covered herein are those where pulse echo straight beam ultrasonic probes are used to produce longitudinal wave for the detection of flaws. The signal available for processing is assumed to be in the form of an A-scan data. This approach is used primarily for the detection of flaws that are perpendicular / orthogonal to the beam or only slightly inclined typically less than $3^{\circ}$ deviation [23]. Angle beam testing is usually used for the detection of inclined flaws.

The A scan signals are assumed to be used directly as features for the ANN training and assessment in the case of $M 1$ and in the other case, $M 2$, parameters based on wavelet decomposition approximation coefficients of the signals were used as features for the ANN. The application of wavelets was carried out to assess signal data downsampling possibilities and noise elimination benefits.

In general the two approaches used for the analysis have produced excellent to very good correlation between predicted and target values. Amplitudes were more accurately predicted than the phase shift which represented the distance between the defects. This observation applies to both the pure signal data and the signal data with noise cases.

It is helpful to highlight the limits of the analysis carried out in this paper. The paper has considered only two defects in close proximity. The focus has been on the use of ANN and wavelets methods to interprete the signal obtained from the flaws. Other problems such as effect of scattering, mode conversion and secondary signals are not covered. Some of these problems are focus of other studies [14]. In reality there could be more than two defects in close proximity in a component and this case will be considered in future work. The paper has 
demonstrated numerically from simulated pulse echo data that ANN and wavelets methods can assist with the interpretation of signals arising from close defects. The data used especially in the cases with noise added to the signal are comparable to experimental data as highlighted in section 4.3 .

\section{Conclusions}

The paper demonstrates the potential of two ANN - wavelets methods to assist with the characterisation two close defects in terms of their sizes and the distance between them. The results from the methods show very good correlation between predicted and target values. Results for the amplitudes were generally better predicted than distance between the defects. Good correlation was also obtained in the cases with noisy signal data. As to be expected filtering to ameliorate the effect of noise in signals assisted good prediction. Filtering the signal before using the wavelet method did not produce better results than direct application of ANN on the filtered data.

\section{References}

1. Zhang, G, Harvey, D M and Braden, D R (2007), "Microelectronic package characterisation using scanning acoustic microscopy", NDT\&E International, 40, 609617.

2. Halmshaw, R. (1991). Non-destructive testing. London, Edward Arnold.

3. Herrera, R H, Orozco, R and Rodriguez, M (2006), "Wavelet-based deconvolution of ultrasonic signals in destructive evaluation", Journal of Zhenjian University SCIENCE A, 7(10), 17481756. 
4. Windsor, C. G., Anselme, F., Capineri, L. and Mason, J. P. (1993). "Classification of weld defects from ultrasonic images. A neural network approach." British Journal of Non-Destructive Testing 35(1): 15-22.

5. Bishop, C M (1996), Neural network for pattern recognition, Oxford University Press, Oxford, U.K.

6. Neural Networks (2011), http://www.statsoft.com/textbook/stneunet.html (accessed September 2011).

7. Igel, C and Husken, M (2000), "Improving the Rprop Learning Algorithm", Proceedings of the Second International Symposium on Neural Computation, NC'2000, pp. 115-121, ICSC Academic Press.

8. The MathWorks, Inc (2010), MATLAB The language of technical computing, USA.

9. Strang, G and Nguyen, T, Wavelets and Filter Banks (1997), Wellesley-Cambridge Press, MA.

10. Addison, P S (2005), "Wavelet transforms and the ECG: a review", Physiological Measurements, 26, R155-R199.

11. Ranja, S (2010), "Exploring the Discrete Wavelet Transform as a Tool for Hindi Speech Recognition", International Journal of Computer Theory and Engineering, 2 (4) 17938201.

12. Lee, $\mathrm{T} \mathrm{H}$, "Wavelet analysis for image processing http://disp.ee.ntu.edu.tw/henry/wavelet_analysis.pdf (accessed November 2011).

13. Press, W Teukolsky, S, Vetterling, W, Flannery, B (1992). Numerical Recipes in Fortran (or C), NY, NY: Cambridge University Press.

14. Lee, K, Estivill-Castro, V, (2010) "Feature Extraction Techniques for Ultrasonic Shaft Signal Classification” 5th International Conference on Computer Sciences and Convergence Information Technology (ICCIT), 366-372. 
15. McKay, M., W. Conover, and R. Beckman (1979), “A comparison of three methods for selecting values of input variables in the analysis of output from a computer code", Technometrics 21, 239-245.

16. Obadiat, M S, Suhail, M A and Sadoun B, (2001), “An intelligent simulation methodology to characterise defects in materials", Information Sciences, (137) 33-41.

17. Lee, K, (2001), "Feature extraction schemes for ultrasonic non-destructive testing inspection", Advances in information sciences and service sciences, (3) 5 125-135.

18. Legendre, S, Massionette, D, Goyette, J and Bose, T, (2001), "Neural classification of Lamb wave ultrasonic weld testing signals using wavelet coefficients", IEEE transactions on instrumentation and measurement, 30, 672-678.

19. Jahankhani, P, Kodogiannis, V and Revett, K (2006), "EEG signal classification using wavelet feature extraction and neural networks”, In: JVA '06. IEEE John Vincent Atanasoff 2006 International Symposium on Modern Computing.

20. Farley, S. (2011), The characterisation of multiple defects in components using artificial neural networks, $\mathrm{PhD}$ thesis, Oxford Brookes University.

21. NDT Systems, http://www.ndtsystems.com/Transducers/DucerCAT-SML.pdf (accessed November 2011).

22. Montgomery, D C, (1997), Design and analysis of experiments, Wiley.

23. Tole, N. M. Tole, Ostensen, H, http://www.isradiology.org/isr/books_basic.php, Chapter 3, (accessed July 2012). 


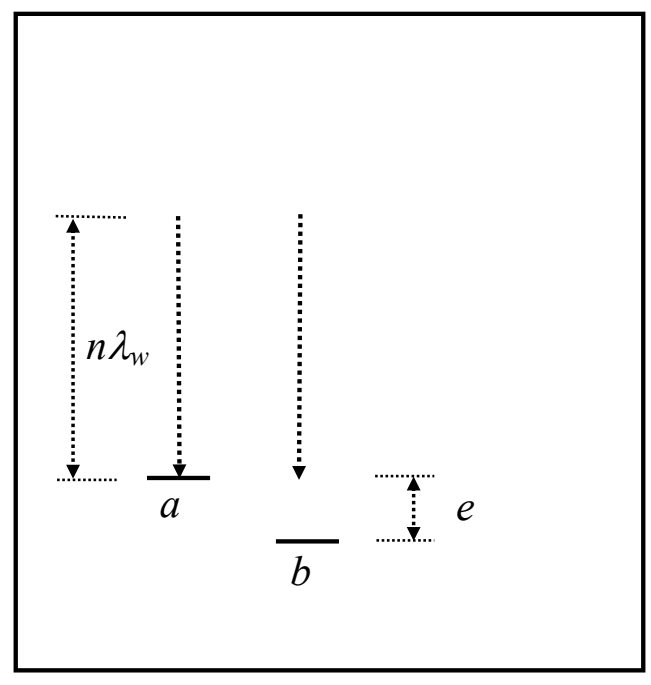

Fig 1

a)

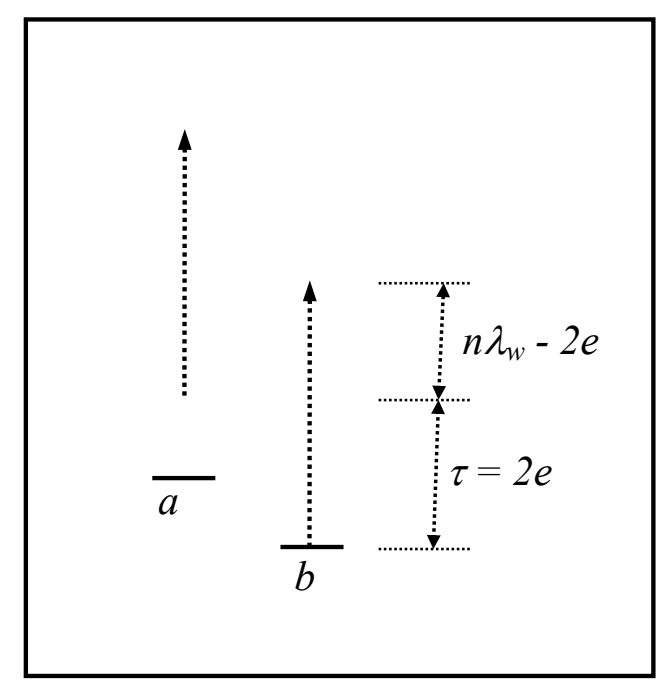

b)

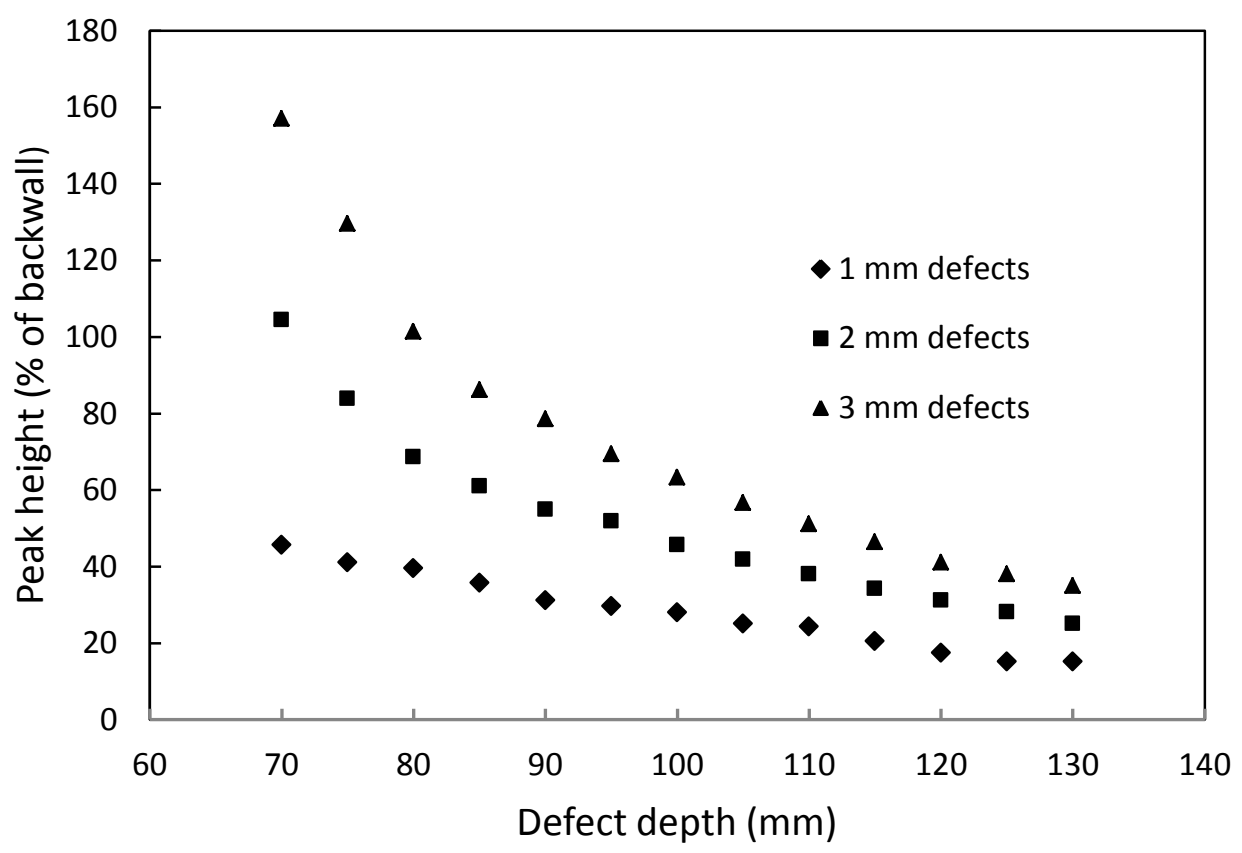

Fig 2 

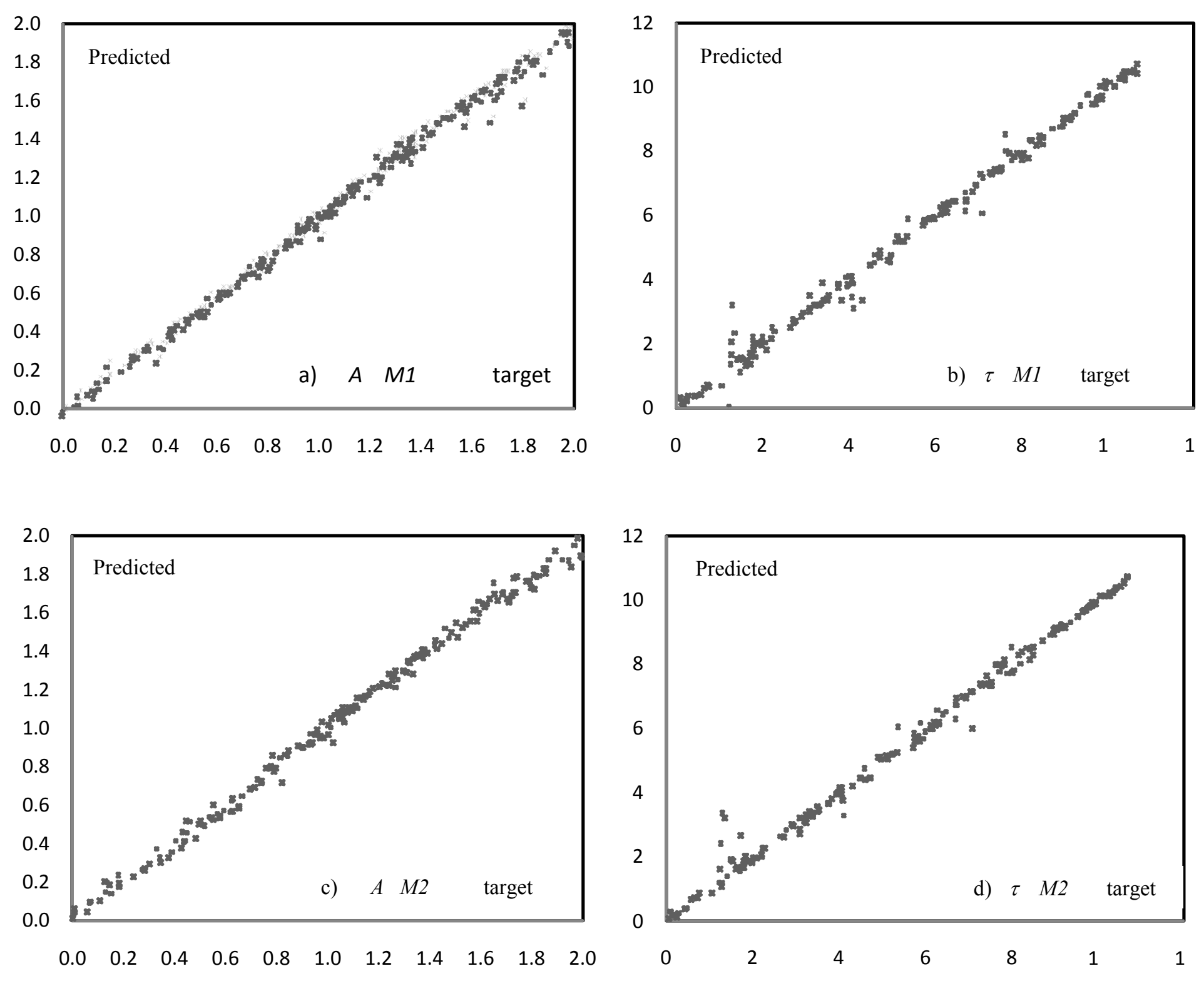

Fig 3

18 


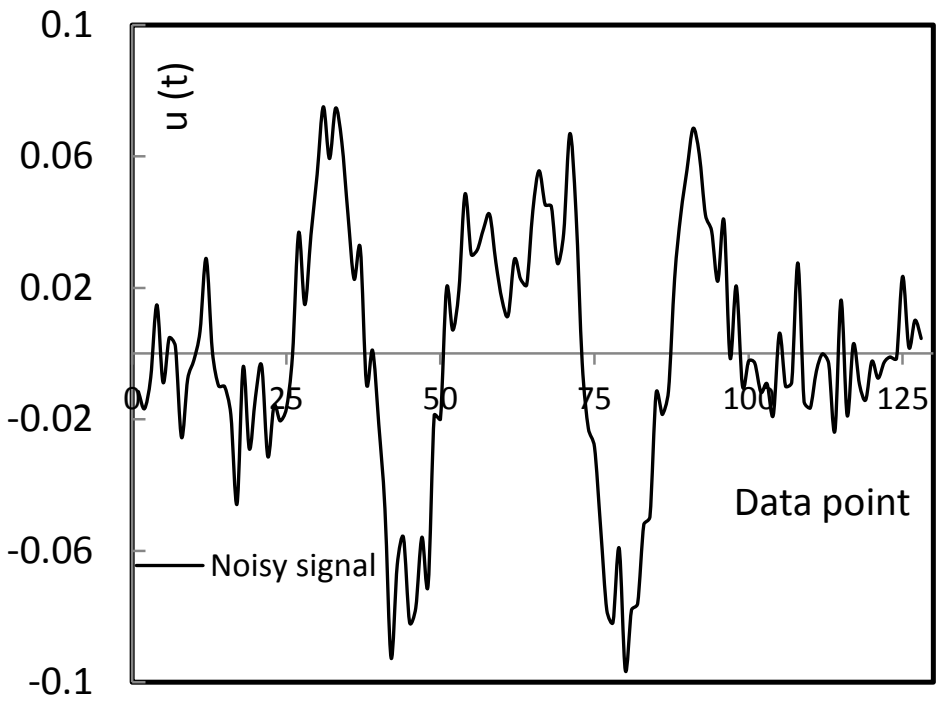

a)

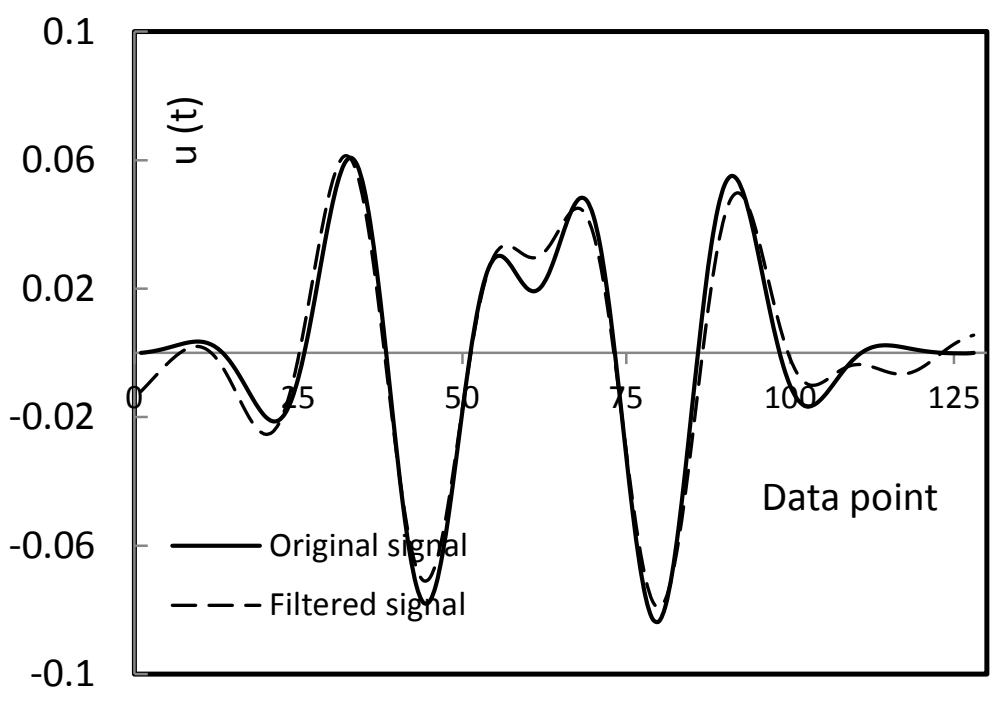

c)

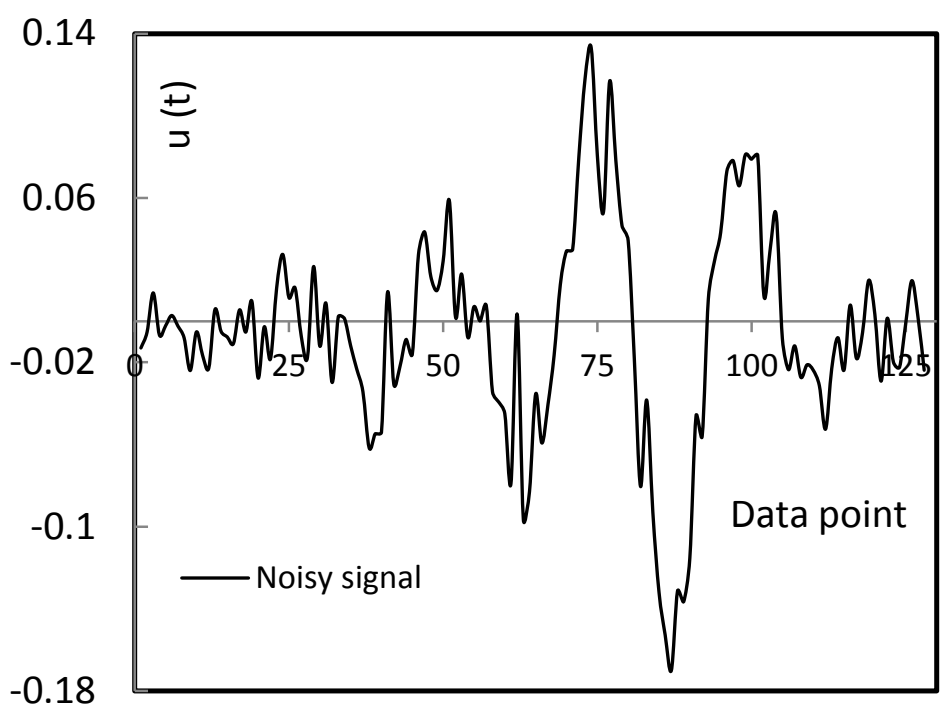

b)

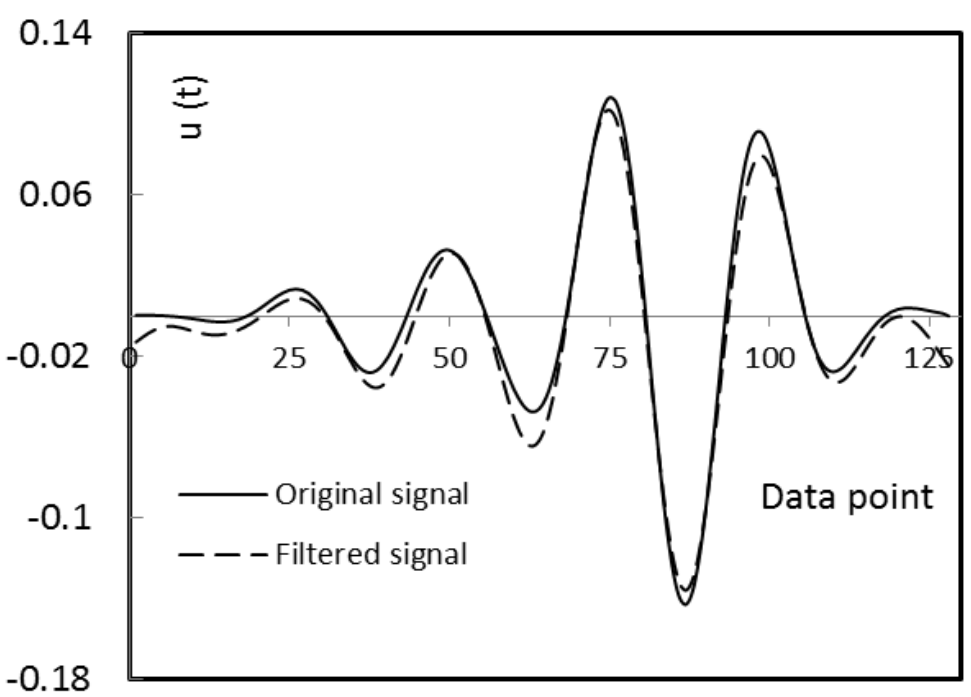

d)

Fig 4 

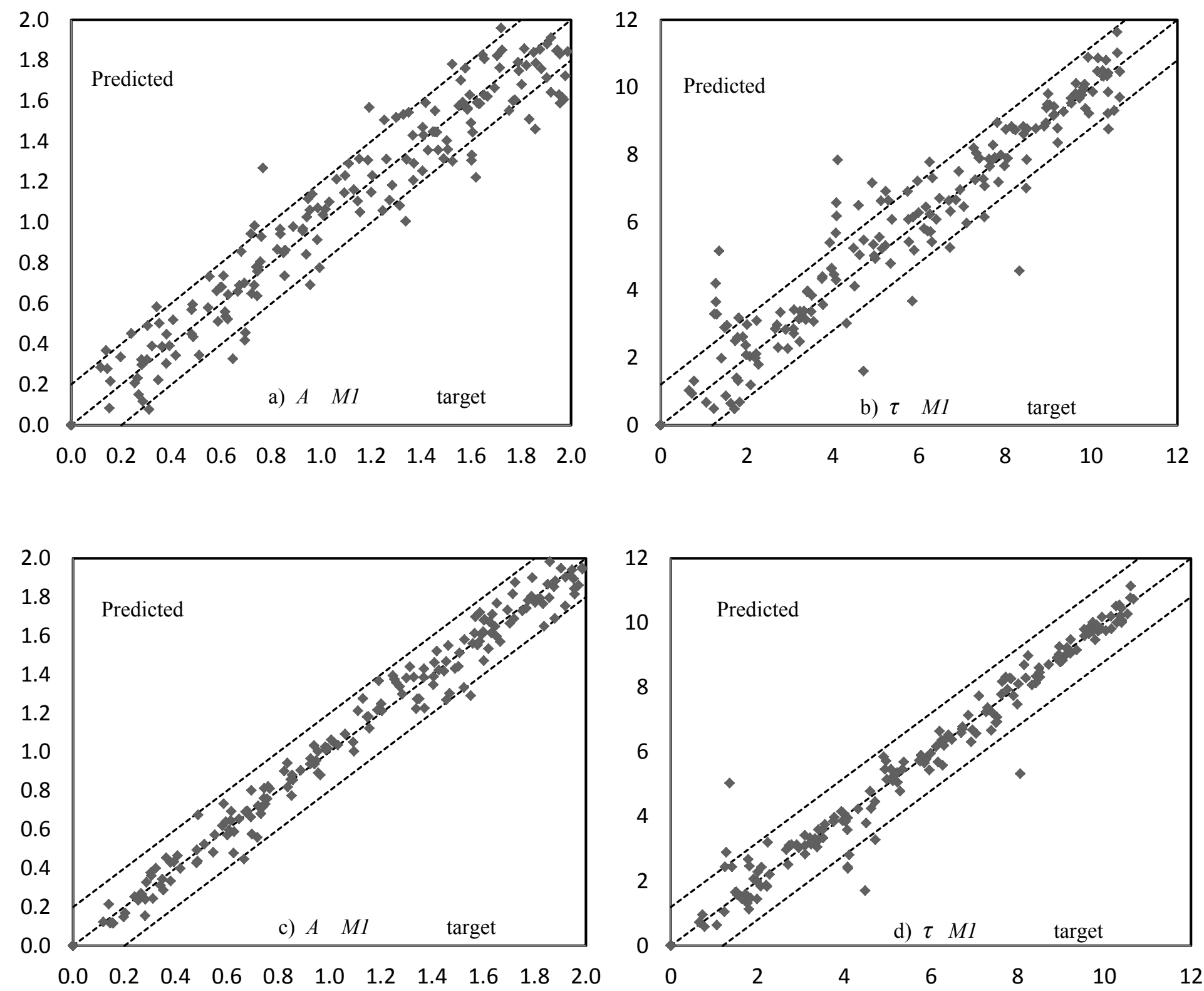

Fig 5

Table 1 (a)

\begin{tabular}{c|c|c|c|c}
\hline \multirow{2}{*}{$\begin{array}{c}\text { Test } \\
\text { Case }\end{array}$} & \multicolumn{3}{|c|}{$R_{X, Y}-M 1$} & $E_{n}(\%)$ \\
\cline { 2 - 5 } & $A$ & $B$ & $\tau$ & All \\
\hline $1\left(A^{+} B^{-} \tau^{-}\right)$ & 0.9996 & 0.9993 & 0.9960 & 1.138 \\
\hline $2\left(A^{-} B^{-} \tau^{+}\right)$ & 0.9985 & 0.9984 & 0.9909 & 1.787 \\
\hline $3\left(A^{-} B^{+} \tau^{-}\right)$ & 0.9995 & 0.9993 & 0.9992 & 0.796 \\
\hline $4\left(A^{+} B^{+} \tau^{+}\right)$ & 0.9989 & 0.9981 & 0.9866 & 1.973 \\
\hline
\end{tabular}




\begin{tabular}{c|c|c|c|c}
\hline \multirow{2}{*}{$\begin{array}{c}\text { Test } \\
\text { Case }\end{array}$} & \multicolumn{3}{|c|}{$R_{X, Y}-M 2$} & $E_{n}(\%)$ \\
\cline { 2 - 5 } & $A$ & $B$ & $\tau$ & All \\
\hline $1\left(A^{+} B^{-} \tau\right)$ & 0.9991 & 0.9988 & 0.9964 & 1.286 \\
\hline $2\left(A^{-} B^{-} \tau^{+}\right)$ & 0.9984 & 0.9970 & 0.9900 & 1.996 \\
\hline $3\left(A^{-} B^{+} \tau^{-}\right)$ & 0.9995 & 0.9996 & 0.9994 & 0.687 \\
\hline $4\left(A^{+} B^{+} \tau^{+}\right)$ & 0.9977 & 0.9974 & 0.9927 & 1.900 \\
\hline
\end{tabular}

Table $1(b)$

\begin{tabular}{c|c|c|c|c|c}
\hline Method & $\begin{array}{c}\text { Signal to } \\
\text { noise ratio }\end{array}$ & \multicolumn{3}{|c|}{$R_{X, Y}$} & $E_{n}(\%)$ \\
\cline { 3 - 6 } & $S_{N R}(\mathrm{~dB})$ & $\mathrm{A}$ & $\mathrm{B}$ & $\tau$ & All \\
\hline$M 1$ & 7 & 0.9515 & 0,9089 & 0.9011 & 8.279 \\
\hline$M 2$ & 7 & 0.9231 & 0.9197 & 0.9219 & 8.568 \\
\hline$M 1$ & 14 & 0.9812 & 0.9593 & 0.9391 & 6.172 \\
\hline$M 2$ & 14 & 0.9579 & 0.9601 & 0.9518 & 7.735 \\
\hline$M 1$ & 21 & 0.9857 & 0.9729 & 0.9663 & 4.811 \\
\hline$M 1$ & 30 & 0.9956 & 0.9866 & 0.9885 & 2.953 \\
\hline
\end{tabular}

Table 2

\section{List of Tables}

Table 1 Product-moment correlation coefficients between target and predicted values $\left(R_{X, Y}\right)$ and the normalised total root mean square error $E_{n}$ for different combinations of the limits of the amplitudes $A$ and $B$ and the phase shift $\tau$ using methods (a) $M 1$ and (b) $M 2$.

Table 2 Product-moment correlation coefficients between target and predicted values $\left(R_{X, Y}\right)$ and the normalised total root mean square error $E_{n}(100 \%)$ for $A$ and $B$ and the phase shift $\tau$ using methods $M 1$, and $M 2$ and different signal to noise ratios. 


\section{List of figures}

Figure 1 Illustration of phase shifting of reflected waves due to different depths of defects a) waves making contact with the first defect b) waves fully reflected from the second defect.

Overlap of reflected waves occur if the distance $e$ between the defects $a$ and $b$ satisfies $e<n \lambda_{w} / 2$.

Figure 2 Distance amplitude correction (DAC) test results for a $200 \times 100 \times 10 \mathrm{~mm}$ mild steel bar with three defect 1, 2 and 3 diameter holes at different depths.

Figure 3 Plots of predicted and target values for amplitude $A$ and phase shift $\tau(\mathrm{rad})$ for different methods $M 1$ and $M 2$.

Figure 4 Two different signals a) and b) with $7 \mathrm{~dB}$ signal to noise ratio and (c) and (d) the corresponding filtered signal and original signal .

Figure 5 Plots of predicted and target values for amplitude $A$ and phase shift $\tau(\mathrm{rad})$ for different signal to noise ratio a) and b) $7 \mathrm{~dB}$ and c) and d) $21 \mathrm{~dB}$. 